eISSN: $2387-1555$

DOI: http://dx.doi.org/10.14201/rea201971326

\title{
LA CONSUMIDAD: VIDA COTIDIANA, CONSUMO Y ESPACIO RURAL ${ }^{1}$ The consumpnity: everyday life, consumption and rural space
}

\author{
Iñigo GONZÁLEZ-FUENTE \\ Universidad de Cantabria \\ \inigo.gonzalez@unican.es \\ Hernán SALAS QUINTANAL \\ Universidad Nacional Autónoma de México \\ $\triangle$ bsalas@unam.mx
}

Fecha de recepción: 30 de octubre de 2018

Fecha de aceptación: 04 de diciembre de 2018

\begin{abstract}
RESUMEN: Los autores presentan el caso del pueblo de Val'Quirico (Tlaxcala, México). Se trata de un espacio rural turistizado que combina elementos propios de los centros comerciales y de los fraccionamientos privados. En primer lugar, como shopping center, Val'Quirico es un lugar multifuncional que cuenta con un grupo de establecimientos comerciales. En segundo lugar, Val'Quirico es un proyecto inmobiliario que responde a las características de comunidad cerrada en cuanto a que, sobre todo, cuenta con servicios de seguridad y vigilancia, y acceso restringido y controlado. Sin embargo, Val'Quirico es algo más que un nuevo caso de diferenciación social a través de los patrones de consumo de los grupos humanos. Val'Quirico es un pueblo que acaba de nacer: Sobre los restos de una exhacienda, a modo de espacio artificialmente turistizado, se alza un conjunto arquitectónico que simula ser un pueblo de estilo medieval europeo. En este contexto, los autores reflexionan sobre los procesos de segregación socio-espacial, y de mercantilización, privatización y artificialización de la vida cotidiana. Estos procesos tienen como eje transversal que se desarrollan en contextos sociales en los que es imperativa la generación de ingresos y la extracción de plusvalías a través de la disciplina del mercado. A modo de conclusión, se aporta para el debate académico el neologismo de consumidad, un espacio rural turistizado entre el centro comercial y el residencial cerrado.
\end{abstract}

Palabras claves: consumo; comunidad; espacio rural; vida cotidiana; consumidad.

ABSTRACT: The authors present the town of Val'Quirico (Tlaxcala, Mexico). This is a touristized rural space that combines characteristics of the shopping centers and the gated communities. First, as a mall, Val'Quirico is a multifunctional place that includes a group of shops. Secondly, Val'Quirico is a real estate project that responds to the characteristics of a gated community; specially, it has security and surveillance private services, and restricted and controlled access. However, Val'Quirico is more than a new case of social differentiation through the consumption patterns of human groups. Val'Quirico is a town that has just been born. On the remains of an ex hacienda, as an artificially turistized space, an architectural ensemble stands pretending to be a town of medieval European style. In this context, the authors reflect on the processes of socio-spatial segregation, and of commodification, privatization and artificialization of everyday life. These processes are developed in social contexts in which the generation of income and the extraction of capital gain through the discipline of the market is imperative. In conclusion, the neologism of consumpnity is contributed for the academic debate, as a touristized rural space between a shopping center and a gated community.

Keywords: consumption; community; rural space; everyday life; consumpnity.

\section{Introducción}

Ya en 1985, KOWINSKI pronosticaba que, con paso del tiempo, una persona podría «obtener cualquier cosa en un mall, desde diamantes hasta yogurt; (...) donar sangre, apostar, correr, meditar o conseguir un cuarto o un condominio y vivir ahí» (KOWINSKI, 1985: 22). Años más tarde, en los noventa, se generalizaba en México la creación de comunidades cerradas, esto es, espacios residenciales protegidos del exterior por bardas perimetrales, puerta de acceso regulada por guardias privados, etc., orientados a clases medias y altas (MARTÍNEZ BORREGO, LORENZEN y SALAS, 2015: 336).

\footnotetext{
${ }^{1}$ Este artículo es producto del proyecto titulado «Estudio etnográfico de pueblos rurales del sur de Tlaxcala especializados en actividades productivas no agrícolas», patrocinado por el PAPIIT UNAM (clave IN 303419)
} 
Sirvan estos dos apuntes para presentar el pueblo de Val'Quirico en el estado mexicano de Tlaxcala. Se trata de un espacio rural que combina elementos propios de los centros comerciales y de los fraccionamientos privados. Sin embargo, Val'Quirico es algo más que un nuevo caso de segregación socioespacial y diferenciación social a través de los patrones de consumo de los grupos humanos. Val'Quirico es un pueblo «que acaba de nacen» (www.mexicodesconocido.com.mx); literalmente, sobre los restos de una exhacienda, a modo de parque temático, se alza un conjunto arquitectónico que simula ser un «pequeño pueblo» de estilo medieval europeo (www.valquirico.com). En el caso de Val'quirico, además, las tiendas ofrecen productos elitistas como comida sudafricana, helados gourmet, habitaciones de lujo, obras de arte, clases de equitación, un automóvil Jaguar y hasta la posibilidad de estudiar una licenciatura en desarrollo y negocios inmobiliarios (http://delfabbrouniversidad.com/).

En este contexto, a partir del fenómeno de Val'Quirico, los autores nos proponemos reflexionar sobre los procesos de segregación socio-espacial (espacios fragmentados para la educación, el trabajo y el consumo) y de mercantilización, privatización y artificialización de la vida cotidiana que actualmente está experimentando la población mexicana. El artículo está estructurado en cuatro apartados principales.

El primero presenta el diseño metodológico de la investigación y unos breves apuntes a modo de contextualización socio-histórica sobre el municipio, Natívitas, en cuyos límites está ubicado el pueblo de Val'Quirico. A continuación, se da paso a las definiciones de consumo, espacio y territorio, y la articulación entre espacio y consumo. En seguida se presentan datos sobre Val'Quirico, a partir de los cuales se aporta para el debate académico el neologismo de consumidad (consumpnity) ${ }^{2}$, un espacio rural artificialmente turistizado entre el shopping center (consumption) y el residencial cerrado (gated commnunity).

\section{Diseño metodológico}

Las reflexiones presentadas son resultado de estudios que se desarrollan en el estado de Tlaxcala (México) desde 2009, los cuales han contribuido a entender la relación local-global desde la perspectiva de las transformaciones sociales, a partir de la experiencia de las poblaciones. La investigación se desarrolla bajo una metodología característica de la antropología y las ciencias sociales que combina diversas técnicas de trabajo de campo, información etnográfica y estudio de caso.

Durante su transcurso, hemos establecido vínculos estrechos con familias cuyos miembros tienen la residencia en varias comunidades pertenecientes al municipio de Nativitas, donde hemos residido en las temporadas de campo. Específicamente, los resultados del presente artículo se han registrado entre 2016 y 2018. Hemos visitado el pueblo de Val'Quirico en varias ocasiones y entrevistado a diferentes actores, residentes, turistas y trabajadores.

\section{Nativitas, Tlaxcala.}

El municipio de Nativitas está ubicado en el Altiplano central mexicano, al sur del estado de Tlaxcala. Comprende una superficie de $61 \mathrm{~km}$. cuadrados, lo que representa 1’52\% del total del territorio estatal, el cual asciende a $4.060 \mathrm{~km}$. cuadrados (INEGI, 2001). Si bien hacia 1950 el porcentaje de población rural de México

${ }^{2}$ Los autores agradecen a la Dra. Paola Velasco Santos (Instituto de Investigaciones Antropológicas - Universidad Nacional Autónoma de México) su participación en el debate sobre la presente investigación y, específicamente, la aportación explícita del concepto de Consumidad. Asimismo, agradecemos a la Dra. Marta Gómez Martínez (Dep. Filología - Universidad de Cantabria) la traducción del neologismo al inglés. 
era 57\% y en el estado de Tlaxcala 61\% (DGE, 1950), para el año 2010 este porcentaje en el país había descendido al 28\%, mientras en Tlaxcala el porcentaje se redujo únicamente a 55\% (INEGI, 2010b). Nativitas es un ejemplo de ruralidad. Sus tierras fértiles por sus condiciones agroclimáticas, de suelos y la disponibilidad de agua de lluvia, ríos y lagunas, se han prestado desde épocas prehispánicas para la agricultura. La población del municipio ha experimentado un ligero aumento en las últimas décadas. Entre los años 1990 y 2010 pasó de 21.485 a un total de 23.621 (INEGI, 1991), de la cual 48\% son hombres y 52\% mujeres (INEGI, 2010a). La proporción de mujeres ha aumentado de acuerdo con la disminución de las actividades agrícolas y el consecuente cambio de actividades, donde los hombres han tendido más a la migración de tipo laboral hacia la Ciudad de México, Puebla o los países del norte (EUA y Canadá).

$\mathrm{Al}$ recorrer los diferentes pueblos que conforman el municipio, se puede constatar que las viviendas, aunque concentradas, están rodeadas por los campos de cultivo y las áreas reservadas a la crianza de animales. La vida cotidiana gira en torno al mercado, las pequeñas actividades comerciales, la molienda del maíz y el transporte de pastura, leña o carbón. Como en los pueblos campesinos, desde la madrugada, los agricultores se encargan del regadío, de cuidar sus parcelas, de la cosecha, de alimentar sus animales, dependiendo de la etapa del ciclo agrícola. Las actividades urbanas se concentran alrededor del edificio municipal, junto a las escuelas y algunas oficinas públicas y entidades financieras. El tráfico por la carretera que atraviesa el municipio conecta los distintos pueblos con San Martín Texmelucan, Puebla y Tlaxcala, las tres ciudades más recurridas por los nativitenses en busca de servicios y mercados.

En el año 2010, la Población Económicamente Activa - PEA del municipio de Nativitas era de 8.062 personas, de la cual 30'8\% se dedicaba a actividades primarias (agricultura por cuenta propia, jornaleros y unidades de producción familiar), contrastando con la PEA del estado en estas actividades que es 15’6\% (INEGI, 2010a); 25’5\% se dedicaba a actividades secundarias, ocupados de obreros y empleados en industrias manufactureras; y 43’5\% a actividades terciarias en servicios comerciales, negocios por cuenta propia, empleados en servicios educativos, en la administración pública, en hoteles y restaurantes (INEGI, 2010a). En cuanto al nivel de ingresos, casi 35\% ganaba entre uno y dos salarios mínimos y casi 70\% menos de dos; tan sólo 4’1\% ganaba más de cinco salarios mínimos mensuales (INEGI, 2003)³.

La información recabada en el trabajo de campo pone en evidencia un patrón laboral de las familias. Cada una se caracteriza porque alguno de sus miembros trabaja en las industrias cercanas, cuentan con un pequeño comercio, continúan cultivando para el autoconsumo y criando animales de traspatio, y realizan múltiples actividades centradas en generar ingresos monetarios como vender lonches, lavar y planchar ropa, cuidar niños, manejar un taxi, participar en el servicio doméstico, labores de limpieza, etcétera, a lo que se ha venido a sumar ahora la oferta de empleos existente en Val'Quirico. La ruralidad actual que viven los nativitenses más jóvenes es diferente a las experiencias de los ejidatarios y campesinos en el pasado reciente, cuya principal actividad era cultivar la tierra. Además, la fragmentación industrial ha reducido el trabajo formal y estable, el cual ha sido sustituido por otras formas de trabajo y contrataciones, con bajos salarios, la frecuencia del autoempleo, labores informales y actividades móviles.

\footnotetext{
${ }^{3}$ El salario mínimo vigente en México a partir del 1 de enero de 2016 es de $\$ 80.04$ pesos al día (SAT, 2017). En el intervalo de la investigación (2016-2018), un dólar estadounidense equivalía entre 17 y 20 pesos mexicanos.
} 


\section{Consumo}

El punto de partida del presente epígrafe es la estrecha relación entre desigualdad social y prácticas de consumo. El consumo es una expresión de la posición en la estructura social. En una sociedad capitalista de mercado, lo que se compra, cómo se compra y dónde se compra tiene una relación directa con la capacidad económica de las personas (SARAVÍ, 2015: 193). Por ejemplo, llevar una bolsa original de Dolce \& Gabbana no es lo mismo que llevar una bolsa de imitación o clonada; la primera se compra en una tienda oficial ubicada en un centro comercial y la segunda en un mercado popular; comprar la primera implica un estilo de vida que depende de un fuerte poder adquisitivo y comprar la segunda implica un estilo de vida propio de la precariedad e informalidad.

Ahora bien, la relación del consumo con la clase social no es unidireccional, sino más bien se trata de una relación recíproca. El consumo no solo refleja las desigualdades de clase, sino que las construye y establece. En cuanto a las especificidades y contrastes entre los estilos de vida de los sectores más privilegiados y los menos favorecidos, el consumo supone posibilidades y oportunidades para los primeros (para quienes se ha naturalizado el paso del consumo al consumismo), y restricciones y constreñimientos para los segundos. Específicamente, el consumo se organiza a partir del eje formalidad-informalidad. Por un lado, los sectores privilegiados experimentan mercancías originales - las marcas- obtenidas en centros comerciales. Por otro lado, el sector informal (tianguis ${ }^{4}$, mercadería ilegal) representa para los sectores populares la principal vía de acceso a los bienes materiales y culturales.

En este punto, resulta extremadamente interesante profundizar en la relación dialéctica entre consumo y producción, en el sentido de que existe una articulación directa entre la condición precaria del trabajo y las dinámicas y posibilidades de consumo de la población. VELASCO (2017) informa sobre la relación entre las condiciones de vida y trabajo en la subindustria del pantalón -los jeans- y el consumo de mercancías baratas de imitación de las marcas originales-. Por un lado, la inestabilidad de los pagos, la ausencia de prestaciones, la flexibilidad horaria, la desprotección sanitaria (ciertas tareas del proceso de producción de los jeans implican la manipulación directa de químicos nocivos para la salud) son las características de las condiciones laborales de la población.

Por otro, esta flexibilidad y precariedad laboral permite el abaratamiento de los costos y, consecuentemente, posibilita un consumo de bienes y servicios (teléfonos, zapatos, ropa, accesorios a bajo coste) a trabajadores igualmente precarios. En palabras de VELASCO (2017: 42), «la producción pirata, ilegal y precaria posibilita el consumo de una multitud proletaria y es el motor de un circuito de subsidio mutuo que ha contribuido a reconfigurar a estos sujetos $[\ldots]$ como neoliberales».

Desde el punto de vista subjetivo, el consumo ofrece una amplia gama de experiencias que permiten profundizar en cómo viven los sujetos la desigualdad desde sus propias experiencias, sentidos, emociones, reflexiones, expresiones, etcétera. SARAVÍ (2015: 39-51) entiende que se puede hablar de al menos tres categorías de la dimensión subjetiva de la desigualdad: la cultural, la social y la propiamente subjetiva. La dimensión subjetiva cultural refiere al repertorio cultural que consideramos de «sentido común», de supuestos inconscientes, que se ven como naturales, «normales». La dimensión subjetiva social refiere a la desigualdad que es producida y reproducida, de manera inadvertida, por los propios individuos a través de sus relaciones sociales cotidianas. Esta dimensión incluye prácticas como hacer determinados amigos ya sea en el club privado, ya sea en el barrio,

${ }^{4}$ Tianguis, palabra de origen náhuatl que proviene de tianquiztli que significa sitio para vender, comprar o permutar (MONTEMAYOR, 2007); se utiliza como el mercado que se realiza al aire libre un día específico de cada semana. 
o casarse con determinadas personas. La dimensión propiamente subjetiva estaría ligada a la emotividad y reflexividad del individuo.

Entonces, siguiendo a este mismo autor (SARAVÍ, 2015: 23), el consumo de bienes y servicios, y los estilos de vida tienen la capacidad de construir murallas o abrir puertas. Por un lado, los bienes y actividades que son objeto o contexto de consumo fungen como fronteras que delimitan los estilos de vida. Por otro lado, las experiencias de consumo construyen y reproducen espacios socioculturalmente homogéneos, los cuales generan distanciamiento social ya sea por ajenidad, incompatibilidad o desconocimiento del otro (SARAVÍ, 2015: 220).

En cuanto a la dimensión subjetiva cultural, las prácticas de consumo se van asumiendo como naturales por parte de las propias personas. Así, el estilo de vida no se construye únicamente por ciertos bienes o marcas. Incluye toda una serie de aspectos que expresa una condición de clase (dimensión objetiva) pero que también «va nutriendo la vida y experiencia cotidiana para constituirse finalmente en la práctica de una condición de clase» (SARAVÍ, 2015: 198).

Estos aspectos van desde una concreta forma de hablar - con ciertas palabras, formas de saludar, expresiones y actitudes- hasta las actividades que ocupan el tiempo de ocio. Procesos tan simples como la soltura en determinados contextos, las diferencias de gustos y modales, son rutinas que reproducen la jerarquía y la distancia social. Es más, esta distancia no sería en principio negativa si no fuera porque se encuentra atravesada por una profunda desigualdad (SARAVÍ, 2015: 223). Por ejemplo, un estudiante menos privilegiado puede acceder a través de becas a centros privados de prestigio. Sin embargo, a la hora de generar relaciones entre los más privilegiados y los menos privilegiados, estos pueden pagar la colegiatura y «estar» pero no podrán pagar y compartir el estilo de vida, el shopping en EE.UU, las vacaciones en Cancún, las salidas a los restaurantes mejor valorados, etcétera. La dimensión subjetiva social incide en el significado social que ciertos bienes tienen en relación con las personas con las que se comparte cotidianidad. Por ejemplo, los espacios y actividades de ocio difieren sustancialmente entre las personas más y menos privilegiadas económicamente hablando. Mientras las primeras acuden al cine usualmente en el contexto de centros comerciales, los sujetos pertenecientes a las clases populares acuden en contadas ocasiones, no lo hacen o cuando lo hacen es solamente para dar un paseo.

Esta dinámica ocurre de manera más observable en las ciudades, donde conviven clases sociales muy diferenciadas. En las zonas rurales existen menos lugares de consumo y, por ejemplo, en Nativitas, no hay centro comercial ni cine, y sus habitantes deben acudir a las ciudades cercanas. En el caso estudiado, Val'Quirico representa la experiencia de centro comercial y residencial en el medio rural.

Finalmente, en cuanto a la dimensión propiamente subjetiva, se puede señalar que el consumo es importante para todos los sectores sociales. Los estímulos del consumo a través de la publicidad y los medios de comunicación llegan a todos los sectores. Sin embargo, mientras para los menos privilegiados, el acceso limitado a bienes y servicios conlleva un sinfín de frustraciones, los más favorecidos experimentan disfrute tanto del consumo físico (un viaje) como el hecho de compartirlo (con tus iguales). En definitiva, la dimensión subjetiva del consumo (generación y reproducción de fronteras simbólicas) es esencial para hacer posibles distancias y diferencias materiales, económicas y políticas, constituyéndose así «una poderosa herramienta de legitimación de la estructura social desigual» (SARAVÍ, 2015: 207). 


\section{Espacio y territorio}

Con fines operativos, en este artículo los autores entendemos espacio en su acepción más genérica como la dimensión a partir de la cual se materializan los objetos, los fenómenos o los procesos. La categoría de territorio es mucho más concreta y particular que la de espacio. Refiere a una dimensión de la superficie terrestre, y alude a una adscripción política que no tiene la de espacio. Bajo esta perspectiva, la noción de territorio está vinculada a la diferenciación respecto de lo ajeno, al señalamiento en referencia a quién pertenece y al intruso (LÓPEZ LEVI y RAMÍREZ, 2012).

En este epígrafe, nos vamos a centrar en el espacio (urbano y rural) como expresión más visible y palpable de la desigualdad social. Y lo vamos a hacer en un contexto sociohistórico marcado por la globalización y el neoliberalismo. En términos generales, nos referimos a neoliberalismo como «un estilo de gobierno en donde el Estado es gestionado como si se tratase de una empresa», representando así «el abandono de la gestión pública como mediadora entre el mercado y la sociedad -redistribución- para ser sustituido por una administración cuyo principal objetivo es la competitividad y el crecimiento» (CÓCOLA, 2016: 36).

Específicamente, a partir de los años noventa ${ }^{5}$, la relación público-privado adquiere unas nuevas características: se da una primacía de lo privado sobre lo público que impone o trata de imponer la propiedad privada en todos los sectores, tanto urbanos como rurales (ALVARADO y DI CASTRO, 2013: 15; MARTÍNEZ BO-

RREGO et al., 2015: 316). Es más, la preeminencia de las lógicas neoliberales hace que cualquier intervención en la ordenación y gestión del territorio tenga que ser económicamente rentable y tenga como principales protagonistas a los sectores empresarial y financiero (AA.VV., 2016: 18). Estamos hablando por ejemplo de sustituir la plaza como máxima expresión del espacio público por centros privados de encuentro y socialización; el banco para sentarse por la terraza del restaurante; las tiendas tradicionales (abarrotes, reparación de calzado, tintorería, lavandería, etc.) por los comercios elitistas y pertenecientes a multinacionales y, en definitiva, la vida en la calle, por experiencias tematizadas para el consumo.

Por otro lado, más significativo aún si cabe, estamos hablando de la mercantilización de los ámbitos más importantes de la vida cotidiana, incluidos la habitabilidad, la movilidad y los espacios de sociabilidad; específicamente, hablamos de espacios que ya no contemplan ningún tipo de contacto humano más allá de la relación comercial (hoteles, palacios de congresos, centros comerciales, tiendas de diseño, tiendas de productos exóticos, galerías de arte, apartamentos turísticos); donde la cohesión social y la participación ciudadana se reducen a un divertido acto de consumo (AA.VV., 2016: 17-8; CÓCOLA, 2016: 41-3).

Desde luego, el proceso de privatización está muy ligado a la incapacidad de los estados de garantizar la seguridad de los ciudadanos. ALVARADO y DI CASTRO (2013: 19) consideran que el incremento de las urbanizaciones cerradas tiene una relación directa con la escalada de la violencia generalizada. PIRES DO RIO (2007: 12) da un paso más y señala que «el habla del crimen» (narrativas cotidianas, comentarios, conversaciones e incluso bromas y chistes sobre el crimen violento) consigue naturalizar «la percepción de ciertos grupos como peligrosos. De modo simplista divide el mundo entre el bien y el mal y criminaliza ciertas categorías sociales».

Este proceso de urbanización y mercantilización tiene varios fenómenos asociados. En primer lugar, hablamos de segregación espacial, esto es, la concentración de población desfavorecida en territorios

${ }^{5}$ En líneas generales, se puede hablar de tres grandes etapas en los patrones de diferenciación social y de separación espacial (segregación): desde finales del s. XIX hasta 1940, el espacio urbano se caracterizó como «ciudad concentrada» y heterogénea; entre 1940 y 1980 se puede hablar de la forma urbana «centro-periferia», de manera que las clases media y alta se concentran en el centro y los clases bajas en los barrios periféricos; finalmente, desde los 90 hasta la actualidad, el principal instrumento de la segregación son los «enclaves fortificados», «espacios privatizados, cerrados y monitoreados, para residencia, consumo, recreación y trabajo» (PIRES DO RIO, 2007: 257). 
caracterizados por su degradación física y social (MARTÍNEZ BORREGO et al., 2015: 316-8). Esto se expresa en la construcción tanto en espacios urbanos como espacios rurales de comunidades cerradas, esto es, espacios residenciales protegidos del exterior por bardas perimetrales, puerta de acceso regulada por guardias privados, etc., orientados a clases medias y altas. Se trata de lugares donde ni siquiera existen banquetas, lo que obliga al uso del automóvil. Aunque reciben la llegada de muchos trabajadores provenientes del «mundo real», apenas hay comunicación y mucho menos convivencia entre los dos mundos.

En segundo lugar, otro de los fenómenos es el de la gentrificación tanto urbana como rural. La gentrificación es el proceso de incursión de población externa en espacios ocupados por «nativos», así como el proceso de incremento de valor de las propiedades y, finalmente, el proceso de expulsión de la población nativa hasta el punto de que se puede hablar de «desplazados» (CÓCOLA, 2016: 45; MARTÍNEZ BORREGO et al., 2015: 317 y 332). Poco a poco, se van rehabilitando calles y viviendas, y los habitantes que no cumplen con el estatus de barrio renovado son expulsados; a su vez, las tiendas tradicionales se van quedando sin clientes ya que los nuevos consumen en centros comerciales. Entonces, estas tiendas van cerrando y son sustituidas por nuevos establecimientos orientados hacia el turismo y personas de mayor nivel adquisitivo (AA.VV., 2016: 20). La gentrificación es sin duda un proceso a través del cual se transforma el espacio público en espacio de consumo, de diversión y espectáculo, en una «máquina de entretenimiento» a través del mismo tipo de elementos: centros históricos peatonales, bares y restaurantes con sus terrazas, actividades culturales, festivales de todo tipo, edificios espectaculares, puertos deportivos, etcétera (CÓCOLA, 2016: 37-8). Finalmente, «el antagonismo entre el barrio entendido como espacio abstracto del que obtener plusvalías y el barrio como lugar habitado es (...) expresión de la lucha de clases» (CÓCOLA, 2016: 54).

Específicamente, se puede hablar de procesos de gentrificación cuando se presentan los siguientes rasgos (o parte de ellos) en distintos grados: reinversión de capital a través del cambio en los usos del suelo; incremento de población de mayores ingresos; cambios en el paisaje; confluencia de los intereses privados y las autoridades; desplazamiento directo o indirecto de grupos de ingresos menores. En el caso que traemos en estas páginas, Val'Quirico, el uso de suelo ha pasado de ser claramente agropecuario a comercial; la población que visita y reside en Val'Quirico pertenece a clases socioeconómicamente privilegiadas; el paisaje ha mutado de tierra cultivada a construcciones residenciales y comerciales; el proyecto cuenta con todo el apoyo de los gobiernos municipal y estatal; el proyecto de Val'quirico ha duplicado el precio de la tierra sin que todavía se pueda asegurar que ello esté impactando en desplazamientos directos o indirectos de los residentes de las comunidades limítrofes. En este sentido, el plusvalor está afectando de momento únicamente a los precios del suelo agrícola e, igualmente, Val'Quirico podría llegar a atraer y/o retener población debido a los puestos de trabajo que genera, tal y como evidencia LORENZEN (2014) para el caso de Morelos.

Las urbanizaciones cerradas pueden observarse tanto en proyectos privados de empresas inmobiliarias como en las acciones de los propios habitantes. En primer lugar, los condominios cerrados constituyen el tipo de vivienda destinado a las clases altas. Son la versión residencial de una categoría más amplia de nuevos emprendimientos denominados enclaves fortificados, fenómeno que está cambiando la manera en que las personas de las clases media y alta viven, consumen, trabajan y gastan su tiempo de ocio. Estos enclaves son consecuencia de la prevalencia de lo privado y exclusivo frente a lo público y abierto. Las características de estos enclaves son que están demarcados físicamente y aislados por muros, rejas o espacios vacíos. Están volcados hacia el interior y no en dirección a la calle; están controlados por guardias armados y sistemas de seguridad que imponen reglas de inclusión y exclusión. Los enclaves organizan explícitamente la desigualdad social (PIRES DO RIO, 2007: 
313-4) y se alimentan de esta. Mención aparte merece la cuestión de la privatización de la seguridad. Cada vez más, ciertos grupos de personas, desconfiando de los cuerpos policiales públicos, tienen la posibilidad de optar por servicios de seguridad privada (incluidos grupos irregulares o ilegales) e incluso por la justicia privada (acciones policiales extralegales).

Esto choca frontalmente con las ideas de democracia, equidad social y, en general, la expansión de los derechos de la ciudadanía (PIRES DO RIO, 2007: 15). En segundo lugar, el concepto urbanístico cerrado incluye el fenómeno de apropiación privada de bienes públicos, como el cierre de calles, en las que sus habitantes se organizan para cerrar el paso vehicular y/o peatonal, por medio de rejas o barreras y casetas de vigilancia.

Resulta importante recalcar en este punto que la respuesta urbanística cerrada no sólo se presenta en los niveles económicos alto y medio, sino también en niveles más bajos, como los proyectos urbanísticos catalogados de «interés social», con planes de desarrollo donde predominan el hacinamiento de viviendas. RIBBECK (2006), al respecto, ha podido calcular un índice de ocupación para la ciudad de Cuernavaca que habla de un $80 \%$ de edificación en las colonias populares, frente al 60\% de las urbanizaciones de las clases altas y medias; y es suficiente con observar desde las carreteras de salida de la Ciudad de México las poblaciones que se han instalado en cerros y sitios, cuyos «desarrollos inmobiliarios» se caracterizan por el hacinamiento, la carencia de áreas verdes, seguridad y servicios.

Además, cuando nos referimos a estos procesos de segregación socio-espacial y diferenciación social, la dimensión subjetiva se edifica a partir de diferentes ideas o conceptos que tienen los protagonistas. Por un lado, estarían los conceptos asociados a las zonas exclusivas, las cuales se refieren al ofrecimiento y obtención de seguridad, comodidades, privacidad, contacto con la naturaleza, ubicación, plusvalía, cuestiones todas ellas presentes en el caso de Val'Quirico. Específicamente, los usuarios de las zonas exclusivas la perciben como segura y cómoda frente a la inseguridad, marginalidad, violencia, degradación, contaminación, conflictos de la realidad que pertenece al mundo que está afuera. Estas zonas exclusivas no están específicamente concentradas en un solo espacio, pero sí que están en unos límites muy precisos. En el caso de las áreas urbanas, las personas privilegiadas viven la experiencia de la ciudad saltando de un espacio a otro (residencial, escolar, de consumo, etc.) como si se tratara de pequeños islotes sobre un mar de espacios vacíos, vaciada de cualquier experiencia humana que no sea rentable para el mercado (ALVARADO y DI CASTRO, 2013: 20; CÓCOLA, 2016: 41; PIRES DO RIO, 2007: 321; SARAVÍ, 2015: 152). En la investigación llevada a cabo entre 2009 y 2010 consistente en el análisis de contenido de los anuncios de agencias inmobiliarias, ALVARADO y DI CASTRO (2013: 123-4), evidenciaban tres cuestiones que explicaban el auge de las urbanizaciones cerradas en Cuernavaca: sentirse seguro en el lugar de residencia; poseer un estilo de vida asociado a servicios como piscina, casa club, etc.; estar cerca de la naturaleza.

\section{Articulación de espacio y consumo}

Es indiscutible el hecho de que la población se encuentra altamente segregada, con diversos estilos de vida, una gran polarización social y grupos humanos que se distinguen por sus patrones de consumo. En México, como en otros países, las actividades de ocio se han desplazado de los espacios abiertos a entornos cerrados. Por ejemplo, cada vez menos niños juegan en la calle, la gente ya no saca su silla para sentarse a la puerta de su casa y observar quién pasa... ciertos grupos sociales casi ya no utilizan los parques y plazas, de manera que el espacio urbano se va segregando. Todo ello se ha visto fortalecido por la inseguridad pública, la cual ha estimulado la creación de comercios, parques y viviendas en entornos herméticos. Asimismo, se ha promovido una vida más 
hacia los interiores y en el espacio público, donde coexiste la delincuencia con la policía. Y en este contexto, el centro comercial representa una imagen idealizada del mundo contemporáneo. Se presenta con una estructura que imita una calle con tiendas a sus lados, con bancas, faroles y carretas que parecen vendedores ambulantes, pero no lo es. La gente se siente más segura adentro (LÓPEZ LEVI, 2007: 110).

Por el contrario, la población mexicana menos privilegiada no siempre tiene acceso a lo que se vende en los centros comerciales, apoyándose en otro tipo de comercios, el ambulante, el del mercado popular, los productos piratas y aquellos que se encuentran en la economía informal. Tanto la plaza pública como el centro comercial tienen comercio, turismo, esparcimiento e interacción social, pero en el caso de los grandes centros comerciales no se permiten manifestaciones políticas (el visitante no tiene por qué recordar la problemática social ni la suya propia), actividades recreativas ni deportivas.

Además, el medio ambiente está controlado en cuanto a temperatura, precipitación, calidad del aire, basura, etcétera. Así con todo, los centros comerciales han sido permeados por todos, de manera que representan un espacio social fragmentado, son una manifestación del poder y su espacio refleja una cultura dominante (LÓPEZ LEVI, 2007: 120).

\section{El caso de Val'Quirico}

El pueblo de Val'Quirico, inaugurado el 15 de septiembre de 2014, es un espacio rural que combina elementos propios de los centros comerciales y de los fraccionamientos privados. En primer lugar, como shopping center, Val'Quirico es un lugar multifuncional que cuenta con un grupo de establecimientos comerciales y en el que «las actividades se encuentran entrelazadas unas con otras y van encaminadas a un objetivo común: las ganancias monetarias y el buen funcionamiento económico del lugar para sus dueños e inversionistas» (LÓPEZ LEVI, 2007: 118). Específicamente, Val'Quirico cuenta en el momento de cerrar este artículo (finales del 2018) con 31 tiendas comerciales distribuidas del siguiente modo: 12 restaurantes, 4 bares-cafeterías, 2 hoteles, 2 comercios de artesanías exclusivas; 2 comercios de abarrotes de tipo gourmet (procedentes de otros países distintos a México, por ejemplo, anchoas españolas), 1 joyería, 1 galería de arte, 1 concesionario de automóviles de alta gama, 1 negocio de juegos infantiles, 1 escape-room, 1 negocio de renta de bicicletas y ponys, 1 negocio que ofrece fotografías al momento como si fueran antiguas, 1 tienda de ropa ecológica y, por supuesto, 1 agencia inmobiliaria repartida en varios locales del complejo.

Desde otro punto de vista, en Val'Quirico no existe ningún establecimiento comercial que atienda a las necesidades básicas de alimentación, vestimenta, educación básica y sanidad. Mención aparte merecen la presencia de un centro de equitación y de una Universidad privada que ofrece una Licenciatura en Desarrollo y Negocios Inmobiliarios, además de otros talleres y diplomados relacionados con la industria de la construcción.

En segundo lugar, es un emprendimiento inmobiliario del grupo empresarial Abanza (con sede en Puebla) que responde a las características de comunidad cerrada en cuanto a que, sobre todo, dispone de servicios de seguridad y vigilancia, y acceso restringido y controlado. Finalmente, Val'Quirico comparte con los centros comerciales y con los residenciales cerrados que ambos fueron pensados para estar aislados de su entorno geográfico y social.

Sin embargo, Val'Quirico es algo más que un nuevo caso de segregación socioespacial y diferenciación social a través de los patrones de consumo de los grupos humanos. Val'Quirico es un pueblo que, sobre los restos de una exhacienda, a modo de parque temático, se alza como conjunto arquitectónico que simula ser un «pequeño pueblo» de estilo medieval europeo. Val'Quirico no es un shopping village, un centro comercial que 
simula ser un pueblo (VECSLIR, 2011: 37). Val'Quirico es una comunidad habitacional amurallada, abierta para los turistas, con forma de pueblo toscano y un conjunto de establecimientos comerciales gestionados unitariamente. Val'Quirico es, en definitiva, una consumidad si se juega con los conceptos de consumo (consumption) y comunidad (community), que busca el consumo al mismo tiempo que la convivencia entre los de una misma clase, en un contexto europeo, una experiencia consumible. Desde luego, esta anunciada homogeneización social es absolutamente pretendida, ya que el funcionamiento de la consumidad depende necesariamente de la cercanía de grupos altamente heterogéneos como lo son los trabajadores de los comercios y servicios.

Con consumidad, los autores entendemos que se da un paso más en la conversión del consumo como protagonista indiscutible de la vida cotidiana de las personas. El centro comercial, como decía KOWINSKI, ha permitido y permite obtener cualquier cosa. El fraccionamiento cerrado ha permitido y permite concentrar entre muros a grupos de una misma condición social. La consumidad supone, parafraseando a DURKHEIM, el becho comercial total; supone la mercantilización y artificialización de los ámbitos más importantes de la vida cotidiana, incluidos la habitabilidad, la movilidad y los espacios de sociabilidad. En otras palabras, la consumidad supone el siguiente paso en la apuesta del capital por transformar y/o destruir las formas de vida más populares para convertirlas en prácticas (excluyentes) de consumo. En definitiva, estas transformaciones se enmarcan en el contexto de "progresiva extensión del control y filosofía capitalistas a todos los ámbitos de nuestras vidas» (AA.VV., 2016: 19).

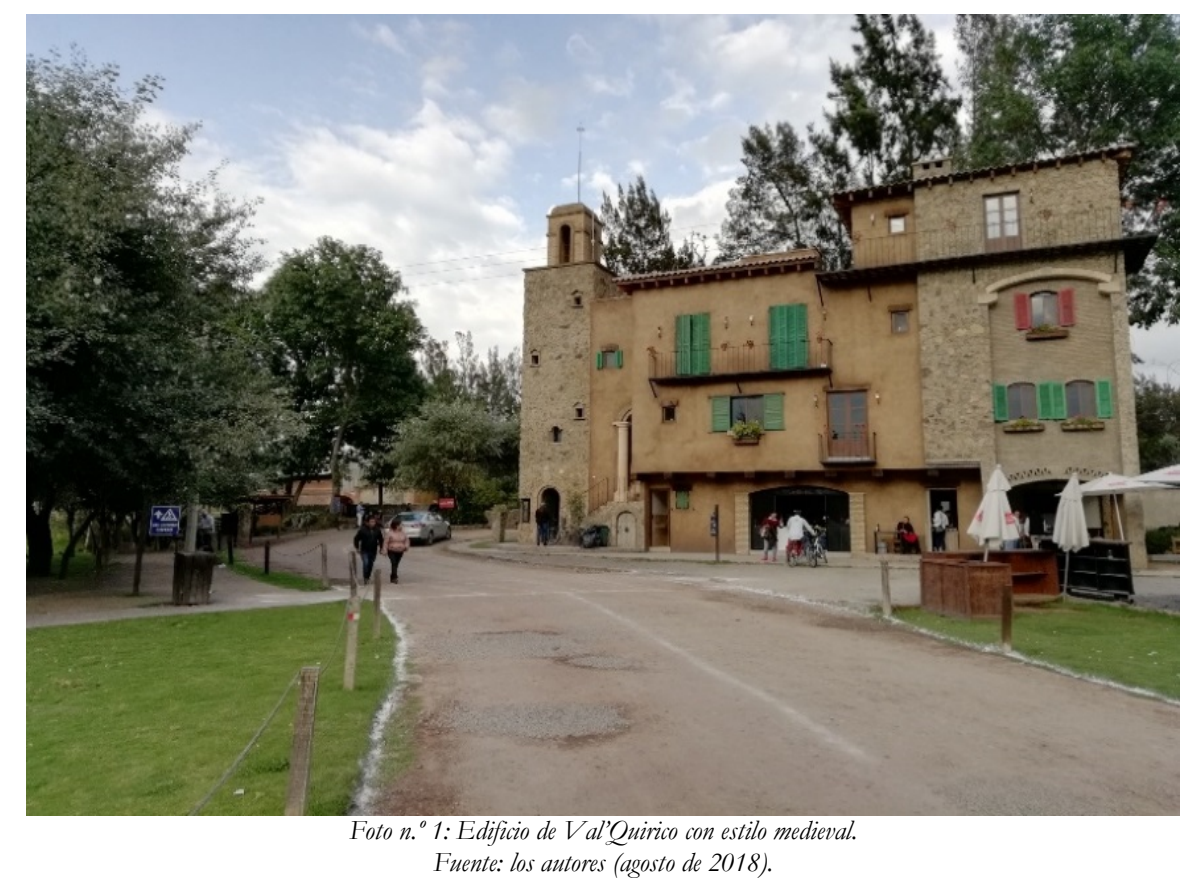

Estamos hablando de espacios que ya no contemplan ningún tipo de contacto humano más allá de la relación comercial; donde la cohesión social y la participación ciudadana se reducen a un divertido y efímero acto de consumo (CÓCOLA, 2016: 41-3).

Hablando de los centros históricos de ciudades como Barcelona, CÓCOLA (2016: 37) coincide en que se ha pasado del consumo en el espacio al consumo del espacio. El autor está pensando en la figura del turista como consumidor dedicado al ocio intensivo por un corto periodo de tiempo. El residente de la consumidad de 
Val'Quirico es ese turista, pero a tiempo completo. El turista de la consumidad es, además de todo lo que puede ser caracterizado como turismo, un (presunto) cliente inmobiliario.

La construcción de la categoría de análisis consumidad permite a los autores reflexionar sobre vida cotidiana y consumo: ¿estamos, como decía KOWINSKI, ante personas que viven en un centro comercial? ¿Es Val'Quirico un parque comercial donde, además, viven y estudian personas? Y si es así, ¿estamos ante la desaparición total de cualquier forma de vida popular (que no genera plusvalía)? Los turistas de Val'quirico no se diferencian mucho de otros sujetos buscadores de ocio y esparcimiento en espacios tematizados para el consumo; sin embargo, los autores consideramos que hay un salto cualitativo muy significativo en pasar de ser un potencial cliente de un trozo de pizza, una prenda deportiva o incluso una obra de arte, a ser un posible comprador de un bien inmueble.

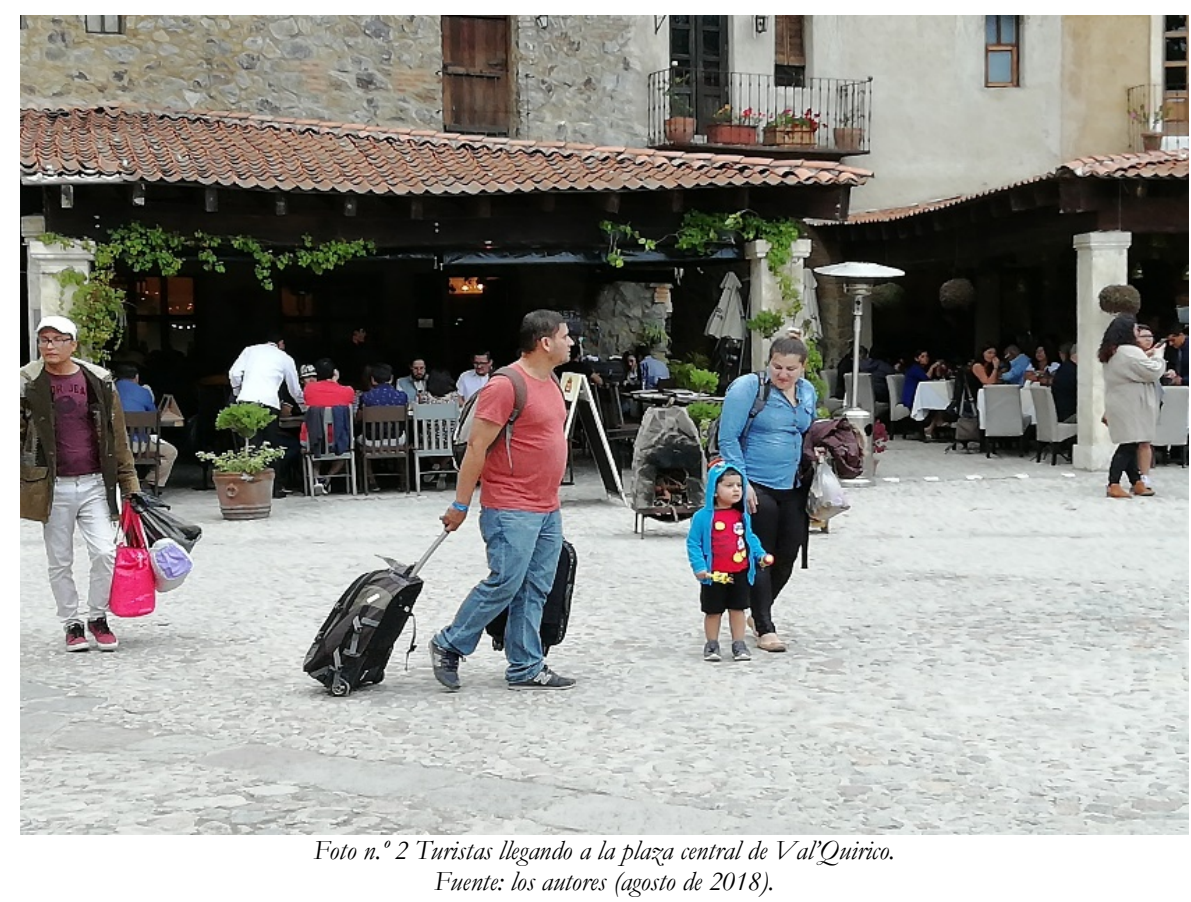

Específicamente, la consumidad sería interpretada por los autores como una nueva manifestación de la cultura dominante: primacía de lo privado sobre lo público, de lo urbano sobre lo rural, de lo individual sobre lo colectivo, del consumir sobre el habitar, de lo tradicional sobre lo moderno.

Estos procesos tienen como eje transversal que se desarrollan en contextos sociales en los que es imperativa la generación de ingresos y la extracción de plusvalías a través de la disciplina del mercado.

\section{A modo de conclusión}

Ha sido el objetivo principal de este artículo reflexionar sobre los procesos que, en cuanto a las intervenciones en la ordenación y gestión del territorio, imponen o tratan de imponer la propiedad privada en todos los sectores, tanto urbanos como rurales.

Es más, la preeminencia de las lógicas neoliberales hace que el territorio tenga que ser económicamente rentable y tenga como principales protagonistas a los sectores empresarial y financiero. 


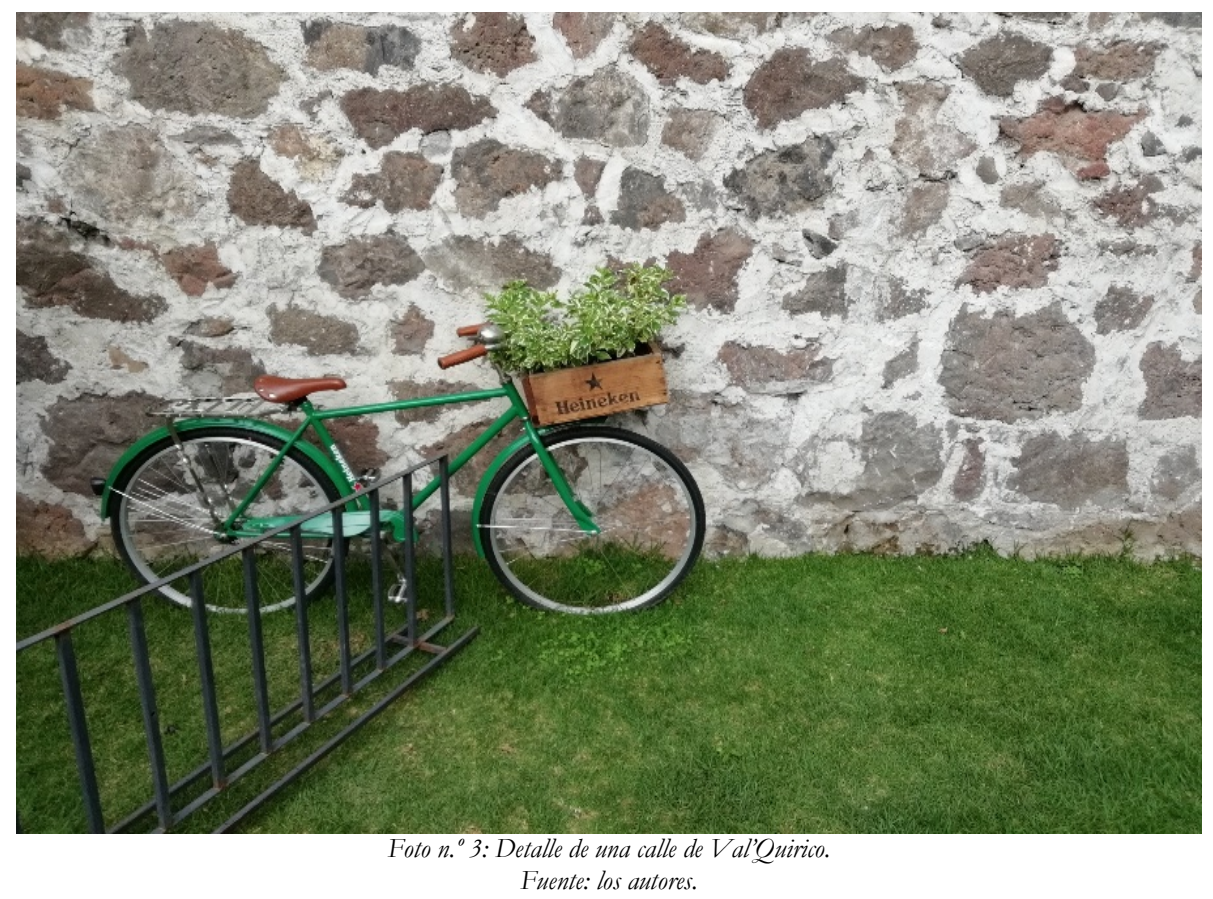

En esta línea, a partir del fenómeno de Val'Quirico, los autores hemos propuesto pensar sobre la mercantilización, privatización y artificialización de los ámbitos más importantes de la vida cotidiana, incluidos la habitabilidad, la movilidad y la sociabilidad, espacios donde la cohesión social y la participación ciudadana se reducen a un divertido acto de consumo.

En este contexto, rescatamos las siguientes ideas. En primer lugar, existe una conexión entre los fenómenos asociados a la transformación socio-espacial y el sistema político-económico global neoliberal.

En segundo lugar, Val'Quirico es un claro ejemplo de que existen procesos de homogeneización sociocultural, especialización turística y artificialización de la vida cotidiana, esto es, de profusión de paisajes banales, homogéneos, que se vinculan a experiencias globales de consumir y habitar, donde los gustos y preferencias, aún de los más privilegiados, se construyen bajo la influencia de los imaginarios de inversores, promotores y constructores. Desde luego, esta pretendida homogeneidad es relativa desde el momento en que el lugar depende de la necesaria participación de sujetos altamente heterogéneos, las clases asalariadas que residen en entornos rurales.

Finalmente, los autores proponemos para el debate académico el neologismo de consumidad (consumpnity), concepto a través del cual pretendemos representar la idea del hecho comercial total, esto es, un espacio rural artificialmente turistizado entre el shopping center y el residencial cerrado, en el que la cotidianidad queda reducida, en la mayoría de las veces, a una relación comercial (en contraposición a la participación ciudadana).

\section{REFERENCIAS BIBLIOGRÁFICAS}

AA.VV. «Introducción». En GRUPO DE ESTUDIOS ANTROPOLÓGICOS LA CORRALA (coord.). (2016) Cartografía de la ciudad capitalista. Transformación urbana y conflicto social en el Estado español. Madrid: Traficantes de sueños, pp. 13-29.

ALVARADO, C. y DI CASTRO, M. R. (2013). Cuernavaca, ciudad fragmentada. Sus barrancas y urbanizaciones cerradas. Ciudad de México: Universidad Autónoma del Estado de Morelos - Juan Pablo Editor. 
CÓCOLA, A. «La producción de Barcelona como espacio de consumo. Gentrificación, turismo y lucha de clases». En GRUPO DE ESTUDIOS ANTROPOLÓGICOS LA CORRALA (coord.). (2016) Cartografía de la ciudad capitalista. Transformación urbana y conflicto social en el Estado español. Madrid: Traficantes de sueños, pp. 31-56.

DGE (Dirección General de Estadísticas) [en línea]. Séptimo Censo General de Población 1950 Tabulados básicos.

Disponible en: http://www3.inegi.org.mx/sistemas/tabuladosbasicos/default.aspx?c=16765\&s=est Consultado: 07/10/2018.

INEGI (Instituto Nacional de Estadística y Geografía) (1991). Tabulados Básicos. Estados Unidos Mexicanos. XI Censo General de Población y Vivienda, 1990. Aguascalientes: INEGI.

INEGI (Instituto Nacional de Estadística y Geografía) (2001). Tabulados Básicos. Estados Unidos Mexicanos. XII Censo General de Población y Vivienda, 2000. Aguascalientes: INEGI.

INEGI (Instituto Nacional de Estadística y Geografía) (2003) [CD-ROM]. Sistema para la consulta de los cuadernos estadísticos municipales. Aguascalientes: INEGI.

INEGI (Instituto Nacional de Estadística y Geografía) (2010a) [en línea]. Censo de Población y Vivienda 2010. Disponible en: http://www.inegi.org.mx/est/contenidos/proyectos/ccpv/cpv2010/ Consultado: 07/10/18.

INEGI (Instituto Nacional de Estadística y Geografía) (2010b) [en línea]. XII Censo General de Población y Vivienda 2010, Tabulados básicos.

Disponible en: http://www3.inegi.org.mx/sistemas/componentes/previsualizador/vista.aspx?arch=/prod_serv/contendos/espanol/bvinegi/productos/estudios/sociodemografico/infantil/2013/702825063672.pdf\&tipo=1 Consultado: 07/10/18.

KOWINSKI, W. (1985). The Malling of America: An Inside Look at the Great Consumer Paradise. New York: William Morrow and Company.

LÓPEZ LEVI, L. «La naturaleza central de los grandes centros comerciales de la Ciudad de México». En VILLARREAL, D. R. y MIGNOT, D. (coord.) (2007) Metropolización, concentración económica y desigualdades espaciales en México y Francia. Ciudad de México: Universidad Autónoma Metropolitana, pp. 107-122.

LÓPEZ LEVI, L. y RAMÍREZ, B. R. «Pensar el espacio: Región, paisaje, territorio y lugar en las Ciencias Sociales». En REYES, M. E. y LÓPEZ LARA, Á. (coord.) (2012) Explorando territorios. Una visión desde las Ciencias Sociales. Ciudad de México: Universidad Autónoma Metropolitana, pp. 21-48.

LORENZEN, M. J. (2014). «La gentrificación rural como factor de persistencia de la población originaria y de las actividades agrícolas: indicios desde Morelos, México». Revista Internacional de Ciencias Sociales, 3(1), 1 14.

MARTÍNEZ BORREGO, E., LORENZEN, M. J. y SALAS, A. (2015). Reorganización del territorio y transformación socioespacial rural-urbana. Ciudad de México: IIS-UNAM - Bonilla Artigas Editores.

MONTEMAYOR, C. (2007). Diccionario de nábuatl en el español de México. Ciudad de México: Universidad Nacional Autónoma de México.

PIRES DO RIO, T. (2007). Ciudad de muros. Barcelona: Gedisa.

RIBBECK, E. «La modernidad informal: colonias populares en la Ciudad de México». En KRIEGER, P. (ed.) (2006) Megalópolis. Ciudad de México: Instituto de Investigaciones Estéticas - Universidad Nacional Autónoma de México / Instituto Goethe / Inter Nations, pp. 203-218. 
SARAVÍ, G. A. (2015). Juventudes fragmentadas. Socialización, clase y cultura en la construcción de la desigualdad. Ciudad de México: CIESAS-FLACSO.

SAT (Servicio de Administración Tributaria) (2017). Salarios mínimos 2016. Secretaría de Hacienda y Crédito Público.

Disponible en:

http://www.sat.gob.mx/informacion_fiscal/tablas_indicadores/Paginas/salarios_minimos.aspx

Consultado: 07/10/18.

VECSLIR, L. (2009). «Nuevas centralidades del ocio y el consumo». Revista Iberoamericana de Urbanismo, 5, 3144.

VELASCO, P. (2017). «Mezclilla, consumo y la configuración de los sujetos rurales neoliberales en Tlaxcala, México». Revista San Gregorio, 18, 35-44. 